\title{
Online GESS: prediction of miRNA-like off-target effects in large-scale RNAi screen data by seed region analysis
}

\author{
Bahar Yilmazel', Yanhui Hu, Frederic Sigoillot ${ }^{2}$, Jennifer A Smith ${ }^{3}$, Caroline E Shamu ${ }^{3}$, Norbert Perrimon ${ }^{1,4}$
} and Stephanie E Mohr ${ }^{\text {* }}$

\begin{abstract}
Background: RNA interference (RNAi) is an effective and important tool used to study gene function. For large-scale screens, RNAi is used to systematically down-regulate genes of interest and analyze their roles in a biological process. However, RNAi is associated with off-target effects (OTEs), including microRNA (miRNA)-like OTEs. The contribution of reagent-specific OTEs to RNAi screen data sets can be significant. In addition, the post-screen validation process is time and labor intensive. Thus, the availability of robust approaches to identify candidate off-targeted transcripts would be beneficial.

Results: Significant efforts have been made to eliminate false positive results attributable to sequence-specific OTEs associated with RNAi. These approaches have included improved algorithms for RNAi reagent design, incorporation of chemical modifications into siRNAs, and the use of various bioinformatics strategies to identify possible OTEs in screen results. Genome-wide Enrichment of Seed Sequence matches (GESS) was developed to identify potential off-targeted transcripts in large-scale screen data by seed-region analysis. Here, we introduce a user-friendly web application that provides researchers a relatively quick and easy way to perform GESS analysis on data from human or mouse cell-based screens using short interfering RNAs (siRNAs) or short hairpin RNAs (shRNAs), as well as for Drosophila screens using shRNAs. Online GESS relies on up-to-date transcript sequence annotations for human and mouse genes extracted from NCBI Reference Sequence (RefSeq) and Drosophila genes from FlyBase. The tool also accommodates analysis with user-provided reference sequence files.
\end{abstract}

Conclusion: Online GESS provides a straightforward user interface for genome-wide seed region analysis for human, mouse and Drosophila RNAi screen data. With the tool, users can either use a built-in database or provide a database of transcripts for analysis. This makes it possible to analyze RNAi data from any organism for which the user can provide transcript sequences.

Keywords: RNAi, Off-target effects, Data analysis, Seed region, miRNA, siRNA, shRNA, High-throughput screening

\section{Background}

RNA interference (RNAi) is a post-transcriptional gene regulatory mechanism [1] that has been widely used for functional genomics studies both in cell lines and organisms. The synthetic duplexes referred to as small interfering RNAs (siRNAs) or short hairpin RNAs (shRNAs) used for RNAi partner with the RNA Induced Silencing

\footnotetext{
*Correspondence: stephanie_mohr@hms.harvard.edu

'Drosophila RNAi Screening Center, Department of Genetics, Harvard Medical School, 77 Avenue Louis Pasteur, Boston, MA 02115, USA

Full list of author information is available at the end of the article
}

Complex (RISC) to target messenger RNAs for degradation in a sequence-specific manner [2,3]. It has been well established that synthetic duplexes have both on-target activities (reducing expression of intended gene) as well as off-target activities (leading to reduced expression of unintended genes) [4]. A significant fraction of falsepositives from RNAi screens is due to off-target effects (OTEs) [5,6]. Many efforts have been made to reduce the number of false positive results due to sequencespecific OTEs, including improved algorithms for RNAi reagent design and incorporation of chemical modifications 
into siRNAs [7]. Another approach has been to develop bioinformatics strategies to identify possible OTEs in screening results.

OTEs have been linked to the mechanism of action of miRNAs, in which a short sequence of bases 2-8 on the $5^{\prime}$ end of a strand of the RNAi duplex (usually the antisense strand), also called the 'seed region', is complementary to the 3' untranslated regions (UTRs) of multiple mRNAs, causing degradation of their associated transcripts $[8,9]$. To improve the interpretation of RNAi datasets and to help minimize follow-up experimental efforts, it is important to identify transcripts that are likely to have scored as a result of seed sequence-based targeting. A number of off-target analysis algorithms have been developed and made available to the scientific community. For example, the Haystack algorithm analyzes RNAi off-target effects based on a predictive model trained with published datasets [10]. The model takes into account four types of seed matches and the length of 3' UTR regions. It requires seed sequence diversity across the dataset and is optimized for large RNAi datasets of sufficient scale with normally distributed scores. Genome-wide Enrichment of Seed Sequence matches (GESS) is another bioinformatics method developed for OTE analysis of RNAi screen datasets [11]. It identifies candidate off-targeted transcripts by investigating the association between matches of the seed regions of RNAi reagents in 3'UTRs with phenotypes observed in largescale screens. In GESS, RNAi reagents are grouped into two categories: siRNA/shRNAs that score in a screen as "with phenotype" or "active", and reagents considered "without phenotype" or "inactive". The algorithm calculates a seed match frequency (SMF) for active and inactive siRNAs/shRNAs for each tested sequence (transcript of a target gene). It is expected that transcripts (and in particular, 3'UTR regions) that are significantly over-represented for seed region matches among active RNAi reagents are more likely to be off-targets. The GESS algorithm has been used successfully to identify off-targeted transcripts in several medium- to large-scale datasets. For example, MAD2 was identified as an off-targeted transcript in a spindle assembly checkpoint components screen and TGF $\beta$-R2 in a screen for novel components of the TGF $\beta$ pathway [11]. GESS was also used in analyzing data from a screen for genes required for homologous recombination and predicted RAD51 as a candidate off-targeted gene; RAD51 OTEs were later confirmed experimentally to be responsible for the activity of many siRNAs identified as hits in the primary screen [12].

Previously, MATLAB was used to program and run the GESS algorithm. Standalone versions of the GESS MATLAB code were provided for several operating systems, including Windows, Linux and Mac. Although it is possible to install and use these stand-alone MATLAB versions, it is not easy for biologists lacking programming and informatics expertise to implement GESS in its original form, as the user has to: 1. download and install the program; 2 . provide files for the reference sequences; and 3. prepare separate files for siRNAs/shRNAs, phenotype and reagent sequence information. In addition, the run time of the program is not optimal for large files. For example, it can take up to 30 hours to analyze 10,000 siRNAs against 27,500 3'UTRs using the standalone MATLAB version of GESS. Hence, an open, user-friendly online tool with improved performance would be of interest to the scientific community.

\section{Implementation}

Online GESS was developed as a Java web application. Twitter Bootstrap 3 front-end framework and jQuery JavaScript library were used to develop the web pages. At the back end, Online GESS contains reference sequences corresponding to 3'UTRs (the region thought to be the most sensitive to miRNA-off-target effects), 5'UTRs, coding sequences (CDS) or full-length transcripts (including non-coding RNAs) in the human, mouse and Drosophila genomes. The human and mouse sequences are obtained from the NCBI RefSeq database. Although these sequences are derived from GenBank records, RefSeq records are non-redundant and have gone through additional levels of validation, annotation, and manual curation. Transcript sequences, as well as CDS and UTR annotations, are retrieved. The Drosophila transcript sequences are obtained from FlyBase (flybase.org) [13], a comprehensive database of Drosophila information that is curated by experts to ensure quality and includes sequences, gene annotation, mutant alleles and publications. Because curation and annotation of reference sequences is an ongoing effort, we have implemented a mechanism for synchronizing reference sequences with each new RefSeq and FlyBase release $[14,15]$.

After a user uploads their annotated screen results (i.e. sequences of active and, if available, inactive RNAi reagents) in Excel, comma-separated values or tab-delimited text format, the online GESS tool extracts the seed sequences from active and inactive RNAi reagent sequences, then searches the transcript sequences for perfect matches. If a set of inactive RNAi reagents is not provided, the program creates a theoretically inactive set by replacing the first nucleotide of each seed region with the complementary nucleotide. The program then calculates the frequencies of matches among active and inactive RNAi reagents, and identifies transcripts that are significantly enriched among active RNAi reagents using the Fisher exact test and Yates chi-square test. When the sample size is small, the $\mathrm{p}$ value from the Fisher's Exact Test is selected; otherwise, the $\mathrm{p}$ value from the Yates Chi Square test is used. Transcripts are then ranked based on 
the selected p-value. Ranks are later used for calculating multiple hypothesis correction. Three multiple hypothesis correction methods are used in the analysis, the Bonferroni, Bonferroni step-down and Benjamini \& Hochberg algorithms, listed in order from most to least stringent correction. Detailed information about the GESS algorithm and analysis methods can be found in the original publication [11].

\section{User interface}

The online GESS application functions as an interface for submitting data and setting parameters for GESS analysis. The output files are sent via e-mail if their size is equal to or smaller than $15 \mathrm{MB}$. For larger files, a link to download resulting files is provided to user by email. The output files will be available for the user to download for 48 hours.

\section{User input}

In order to perform a GESS analysis, the user has to provide siRNA or shRNA information in one of the required formats (e.g. tab or comma separated text file or Excel file). There are two possible layouts for input si/ shRNA files. The first requires the sequences of both active and inactive siRNAs/shRNAs, as well as their corresponding phenotype/activity information (see example file at http://www.flyrnai.org/gess/ActiveAndInactiveSiRNAs.txt). The second layout includes only the sequences of active siRNAs/shRNAs, and phenotype/activity information is not needed (all reagents are assumed to be active; see example file at http://www.flyrnai.org/gess/ ActivesiRNAs.txt). The user then chooses the correct format for their input file by selecting "Input file contains both active and inactive RNAi reagents" or "Input file contains only active RNAi reagents". The user also needs to indicate if the input sequences represent the sense (passenger) or anti-sense (guide) strands of the reagents. In addition, the user has to indicate the reagent type, siRNA or shRNA. If shRNA is selected, it is possible for the user to trim the sequences by one to three nucleotides respectively since sequences provided by the source of shRNA library may not reflect the actual mature siRNA strands that are generated by expected canonical dicer cleavage.

The next step is to specify a reference database. As described above, online GESS has built-in reference databases for the human, mouse and Drosophila genomes. The user can choose one of the three species and then specify the transcript region(s) to search against. The options are 3'UTR (preferred genomic region for GESS analysis), 5'UTR, CDS, full transcript of protein coding genes, or full transcript region of all genes including non-coding RNA. The user can also choose to upload a custom database file. A custom database file should have
FASTA formatted sequences (see example file at http:// www.flyrnai.org/gess/customDatabase.txt). For a customized reference database, the program will search for seed matches along the full length of the sequences provided. If the user would like to focus the search to a specific subregion within a custom reference set, such as 3'UTRs (thought to be the major site of miRNA activity), the user is responsible for uploading only the 3'UTR sequences.

The final step prior to submitting data for processing is to specify any optional parameters. The GESS interface allows users to specify the length of a seed sequence, the minimum number of seed matches to be found in the target sequence, the strand of the RNAi sequence, as well as a statistical threshold value. Currently, the default settings are 7 base pair seed sequence (nucleotides 2-8 from the 5 , end of antisense sequences provided by user), a minimum of one seed match using the anti-sense strand of RNAi only, and a p-value threshold of 0.05 before multiple hypothesis testing correction. The user has the option to perform a control test where each seed sequence of both active and inactive reagents is randomly scrambled. This provides a sense of strength of outliers that may occur at random and more confidence that the significant results are not due to chance. To do this, the user needs to run a parallel test by making corresponding selection under "Advanced Options" at the user interface. This will provide a new set of results and make it possible for users to compare the results obtained for the experimental and control test sets. It is important to note that the program generates only one set of results at a time. Hence, to include a control test in the overall analysis, the control test has to be submitted and run separately.

Online GESS pre-processes the input files and detects mis-formatted records, such as lines missing sequence information, before the analysis starts. If more than $25 \%$ of the records are mis-formatted, the error type (see help page at http://www.flyrnai.org/gess/help.jsp) as well as a few examples will be displayed to the user. This feature enables the user to identify errors in their files immediately and fix them. If less than $25 \%$ of the records fail pre-processing, the tool continues the analysis, ignoring mis-formatted records in the analysis. The user is then informed via email about the number of RNAi reagents that were ignored in the analysis and their location in the file.

\section{Output files}

A GESS analysis generates two output files. The first file lists the transcripts identified by seed region match to active RNAi reagents and their enrichment scores. By default, this file contains results for all tested transcripts. If the user is not interested in getting the full list, the results of significant transcripts can be obtained by choosing "Only Significant Transcripts" under advanced options. When using a built-in database, each transcript is indicated by its 
RefSeq accession number, along with a corresponding gene symbol from NCBI or FlyBase. If a custom database is provided, the comment lines from the FASTA file are displayed. This first file also reports the number of active RNAi reagents that have seed matches to a given sequence, the seed match frequency of active reagents, and the p-values according to both Fisher's Exact and Yates Chi Square tests. The output file also reports the p-value selected for multiple hypothesis correction and the adjusted p-values, as calculated using the Bonferroni, Bonferroni Step-down and Benjamini \& Hochberg methods. Finally, the corrected pvalue thresholds, as well as statistical significance status of each transcript according to each algorithm, are reported in this file. The second file contains the transcript identifiers and a list of active RNAi reagents that match to them. This file contains only the transcripts with $\mathrm{p}$-values $\leq 0.05$. If the analysis fails during input file processing, an email notification is sent to the user (see help page for detailed explanation, http://www. flyrnai.org/gess/help.jsp).

\section{Run time}

The run time of a GESS analysis is dependent upon the input file sizes but in most cases, the analysis is complete within a couple of minutes. For example, in our tests it took two minutes to analyze 10,000 siRNAs against about 68,450 3'UTRs annotated for human genes in RefSeq database (vs61).

\section{Testing}

We compared Online GESS to the standalone MATLAB version using supplementary data from a spindle assembly checkpoint screen as provided in the original GESS publication [11]. The original publication used transcripts from Ensembl as the reference, whereas by default, Online GESS uses transcripts from RefSeq. To do a direct comparison, at Online GESS we uploaded a custom database of 3'UTR sequences from Ensembl as provided in the original publication [11]. We then ran Online GESS using the same parameters as those used in the original publication (a 7 mer seed match from either strand) [11] and obtained the same results. Next, we ran another Online GESS analysis with the same parameters using our built-in database of human 3'UTR sequences (by default, this was the current RefSeq release, i.e. v61). The results were the same at the gene level; that is, MAD2 was the only significant outlier. The only differences we observed between results obtained with the standalone MATLAB version and Online GESS were at the transcript level (not at the gene level) and are attributable to differences in the underlying reference data.

\section{Results and discussion}

Using this tool, we analyzed datasets from several publications (Table 1). For the majority of cell lethality screens, Online GESS did not identify any potential off-target genes when the sequences of top hits were analyzed. Cell lethality is a phenotype that can be triggered by a broad range of biological pathways and it is possible that the GESS approach is not sensitive enough to identify potential off-target genes in these cases. On the other hand, for screens measuring phenotypes with more defined molecular mechanisms, such as a spindle assembly checkpoint components screen and a screen for novel components of TGF $\beta$ pathway, Online GESS identified potential offtarget transcripts (namely, MAD2 in the spindle assembly checkpoint screen and TGF $\beta-R 2$ in the TGF $\beta$ pathway screen). We also analyzed the ionizing radiation (IR) sensitivity screen published by Hurov et al. [16]. The authors report two datasets for the IR sensitivity phenotype. One is comprised of 850 shRNAs that scored in the primary screen; the other comprises 114 shRNAs that were validated using independent shRNAs. Online GESS did not find any potential OTEs among validated hits but found that ZNF480 and SH3BP2, which appear in the primary hit list, might be off-targeted transcripts. This is consistent with the idea that GESS can help narrow down a list of primary hits and prioritize hits for further validation.

In Drosophila, short hairpin RNAs have been used for large-scale in vivo screens [26]. We analyzed results from two screens for embryonic phenotypes associated with maternally loaded shRNAs [24,25]. We did not find any potential off-target genes with either dataset, possibly due to the small size of these studies (1000 or 2300 shRNAs, respectively). As more transgenic shRNA screens are done, we anticipate that GESS will prove useful to detect potential off-targeted transcripts that might be associated with in vivo Drosophila RNAi screen data.

\section{Conclusions}

RNAi is a powerful tool for systematic study of gene functions but results must be analyzed carefully, as screens are associated with false positive and false negative results. Further validation of results, such as screening with multiple independent RNAi reagents, performing $\mathrm{qPCR}$ to verify correlation between knockdown efficiency and phenotypic strength, or RNAi-resistant "rescue" experiments, is time and labor intensive. Detection of potential off-targeted transcripts via automated pre-processing based on our current knowledge of the sources of off-targets, including miRNAlike effects, provides one way to focus limited resources on the most promising candidates. To help support automated detection of off-targeted transcripts in RNAi data, we have implemented a web-based application of seed region analysis for identification of potential off-target transcripts, based on the GESS algorithm. This tool allows users to run 
Table 1 Datasets tested using the GESS online tool and results

\begin{tabular}{|c|c|c|c|c|}
\hline Study & RNAi (species) & Phenotype & $\begin{array}{l}\text { No. active } \\
\text { siRNA }\end{array}$ & $\begin{array}{l}\text { Statistically significant } \\
\text { OT gene }\end{array}$ \\
\hline \multirow{3}{*}{$\begin{array}{l}\text { A genetic screen identifies the Triple T complex } \\
\text { required for DNA damage signaling and ATM and ATR } \\
\text { stability [16] }\end{array}$} & \multirow[t]{3}{*}{ shRNA (hs) } & Ionizing radiation sensitivity & 850 & ZNF480 SH3BP2 \\
\hline & & $\begin{array}{l}\text { Ionizing radiation sensitivity } \\
\text { (validated) }\end{array}$ & 114 & None \\
\hline & & Ionizing radiation resistance & 1080 & None \\
\hline $\begin{array}{l}\text { An intermittent live cell imaging screen for siRNA } \\
\text { enhancers and suppressors of a kinesin-5 inhibitor [17] }\end{array}$ & siRNA (hs) & $\begin{array}{l}\text { Genes involved in spindle } \\
\text { assembly checkpoint } \\
\text { (Dharmacon library) }\end{array}$ & 308 & MAD2L1 \\
\hline $\begin{array}{l}\text { Off-target effects dominate a large-scale RNAi screen for } \\
\text { modulators of the TGF- } \beta \text { pathway and reveal microRNA } \\
\text { regulation of TGFBR2 [18] }\end{array}$ & siRNA (hs) & $\begin{array}{l}\text { Genes involved in TGF- } \beta \\
\text { signaling (Sloan Kettering Inst. } \\
\text { In house library) }\end{array}$ & 409 & TGFBR2 \\
\hline $\begin{array}{l}\text { Genome-wide siRNA screen identifies SMCX, EP400 } \\
\text { and Brd4 as E2-dependent regulators of human } \\
\text { papillomavirus oncogene expression [19] }\end{array}$ & siRNA (hs) & $\begin{array}{l}\text { Genes that contribute to the } \\
\text { repression of the HPV LCR. } \\
\text { (Dharmacon library) }\end{array}$ & 511 & None \\
\hline \multirow[t]{2}{*}{$\begin{array}{l}\text { A genome-wide homologous recombination screen } \\
\text { identifies the RNA-binding protein RBMX as a component } \\
\text { of the DNA-damage response [12] }\end{array}$} & \multirow[t]{2}{*}{ siRNA (hs) } & $\begin{array}{l}\text { Regulator of homologous } \\
\text { recombination } \\
\text { (Dharmacon library) }\end{array}$ & 510 & Rad51 \\
\hline & & $\begin{array}{l}\text { Regulator of homologous } \\
\text { recombination } \\
\text { (Ambion library) }\end{array}$ & 187 & None \\
\hline $\begin{array}{l}\text { Kinase requirements in human cells: I. Comparing } \\
\text { kinase requirements across various cell types [20] }\end{array}$ & TRC shRNA (hs) & $\begin{array}{l}\text { Essential kinases in HeLa } \\
\text { and } 293 \text { T cell lines. }\end{array}$ & 110 & None \\
\hline $\begin{array}{l}\text { A Lentiviral RNAi Library for Human and Mouse Genes } \\
\text { Applied to an Array Viral High-Content Screen [21] }\end{array}$ & TRC shRNA (hs) & $\begin{array}{l}\text { Genes required for mitotic } \\
\text { progression and proliferation } \\
\text { for HT29 cell line }\end{array}$ & 161 & None \\
\hline $\begin{array}{l}\text { Highly parallel identification of essential genes in } \\
\text { cancer cells [22] }\end{array}$ & TRC shRNA (hs) & $\begin{array}{l}\text { Essential genes for } 12 \text { cancer } \\
\text { cell lines }\end{array}$ & 182 & None \\
\hline \multirow{4}{*}{$\begin{array}{l}\text { Systematic investigation of genetic vulnerabilities across } \\
\text { cancer cell lines reveals lineage-specific dependencies } \\
\text { in ovarian cancer [23] }\end{array}$} & \multirow{4}{*}{ TRC shRNA (hs) } & $\begin{array}{l}\text { Essential genes specifically } \\
\quad \text { for Ovarian cell lines }\end{array}$ & $\sim 1500$ (top 3\%) & None \\
\hline & & $\begin{array}{l}\text { Essential genes specifically } \\
\text { for Colon cell lines }\end{array}$ & 1500 (top 3\%) & None \\
\hline & & $\begin{array}{l}\text { Essential genes specifically } \\
\text { for Pancreas cell lines }\end{array}$ & 1500 (top 3\%) & None \\
\hline & & $\begin{array}{c}\text { Essential genes specifically } \\
\text { for Esophageal Squamous } \\
\text { cell lines }\end{array}$ & 1500 (top 3\%) & TACO1 \\
\hline $\begin{array}{l}\text { Depleting gene activities in early Drosophila embryos } \\
\text { with the "maternal-Gal4-shRNA" system [24] }\end{array}$ & shRNA (dm) & $\begin{array}{l}\text { Abnormal embryonic } \\
\text { phenotypes }\end{array}$ & 79 & None \\
\hline $\begin{array}{l}\text { A regulatory network of Drosophila germline stem cell } \\
\text { self-renewal [25] }\end{array}$ & shRNA (dm) & $\begin{array}{l}\text { Abnormal embryonic } \\
\text { phenotypes }\end{array}$ & 329 & None \\
\hline
\end{tabular}

Note: The results presented here were obtained using the following parameters: "3'UTR" as genomic region, "antisense/guide" as strand to use, "7" as seed sequence length. The shRNA libraries from The RNAi Consortium (TRC) were trimmed by $2 \mathrm{bp}$.

off-target analysis with ease. Users can analyze human, mouse or Drosophila datasets directly using built-in reference sequence database. In addition, screen data can be analyzed based on a custom reference database, making it possible to analyze RNAi screen results from any organism and at any scale.

\section{Availability and requirements}

- Project name: Online GESS

- Project home page: http://www.flyrnai.org/gess

- Operating system(s): Platform independent
- Programming language: Server side: Java, JavaScript

- Other requirements: None

- License: Not applicable

- Any restrictions to use by non-academics: None

\section{Abbreviations}

RNAi: RNA interference; siRNA: Small interfering RNA; shRNA: Short hairpin RNA; miRNA: MicroRNA; OTE: Off-target effect; $5^{\prime}$ UTR: Five prime untranslated region; 3' UTR: Three prime untranslated region; CDS: Coding Sequences; NCBI: National Center for Biotechnology Information; TRC: The RNAi Consortium.

\section{Competing interests}

The authors declare that they have no competing interests. 


\section{Authors' contributions}

BY designed the UI, implemented the application, analyzed the datasets and drafted the manuscript. YH guided development and testing, analyzed the datasets and drafted the manuscript. FS developed the original algorithm, contributed to tool evaluation and manuscript editing. JAS participated in the UI design, testing and manuscript editing. CES and NP provided guidance and edited the manuscript. SEM provided oversight, guided development and testing, and helped draft the manuscript. All authors read and approved the final manuscript

\section{Acknowledgements}

The authors would like to thank members of the Perrimon lab and DRSC for helpful suggestions and discussions. In particular, we thank lan Flockhart, Charles Roesel and Arunachalam Vinayagam for help and suggestions. This work was supported by the Dana Farber/Harvard Cancer Center ( $\mathrm{NCl}$ Cancer Center Support Grant \# NIH 5 P30 CA06516) and Harvard Medical School. We also acknowledge NIH funding in support of the Drosophila RNAi Screening Center (R01 GM067761 to N.P.). N.P. is an Investigator of the Howard Hughes Medical Institute.

\section{Author details}

${ }^{1}$ Drosophila RNAi Screening Center, Department of Genetics, Harvard Medical School, 77 Avenue Louis Pasteur, Boston, MA 02115, USA. ²Novartis Institutes for Biomedical Research, Developmental and Molecular Pathways, 250 Massachusetts Avenue, Cambridge, MA 02139, USA. ${ }^{3}$ ICCB-Longwood Screening Facility, Harvard Medical School, 250 Longwood Ave, Boston, MA 02115, USA. ${ }^{4}$ Howard Hughes Medical Institute, 77 Avenue Louis Pasteur, Boston, MA 02115, USA.

Received: 11 February 2014 Accepted: 10 June 2014 Published: 17 June 2014

\section{References}

1. Chapman EJ, Carrington JC: Specialization and evolution of endogenous small RNA pathways. Nat Rev Genet 2007, 8(11):884-896.

2. Boutros M, Ahringer J: The art and design of genetic screens: RNA interference. Nat Rev Genet 2008, 9(7):554-566.

3. Mohr SE, Perrimon N: RNAi screening: new approaches, understandings, and organisms. Wiley interdiscip rev RNA 2012, 3(2):145-158.

4. Jackson AL, Linsley PS: Noise amidst the silence: off-target effects of siRNAs? Trends Genet 2004, 20(11):521-524.

5. Ma Y, Creanga A, Lum L, Beachy PA: Prevalence of off-target effects in Drosophila RNA interference screens. Nature 2006, 443(7109):359-363.

6. Mohr S, Bakal C, Perrimon N: Genomic screening with RNAi: results and challenges. Annu Rev Biochem 2010, 79:37-64

7. Behlke MA: Chemical modification of siRNAs for in vivo use. Oligonucleotides 2008, 18(4):305-319.

8. Sudbery I, Enright AJ, Fraser AG, Dunham I: Systematic analysis of offtarget effects in an RNAi screen reveals microRNAs affecting sensitivity to TRAIL-induced apoptosis. BMC Genomics 2010, 11:175.

9. Anderson EM, Birmingham A, Baskerville S, Reynolds A, Maksimova E, Leake $D$, Fedorov $Y$, Karpilow J, Khvorova A: Experimental validation of the importance of seed complement frequency to siRNA specificity. RNA 2008, 14(5):853-861.

10. Buehler E, Khan AA, Marine S, Rajaram M, Bahl A, Burchard J, Ferrer M: siRNA off-target effects in genome-wide screens identify signaling pathway members. Sci rep 2012, 2:428.

11. Sigoillot FD, Lyman S, Huckins JF, Adamson B, Chung E, Quattrochi B, King RW: A bioinformatics method identifies prominent off-targeted transcripts in RNAi screens. Nat Methods 2012, 9(4):363-366.

12. Adamson B, Smogorzewska A, Sigoillot FD, King RW, Elledge SJ: A genomewide homologous recombination screen identifies the RNA-binding protein RBMX as a component of the DNA-damage response. Nat Cell Biol 2012, 14(3):318-328.

13. St Pierre SE, Ponting L, Stefancsik R, McQuilton P: FlyBase 102-advanced approaches to interrogating FlyBase. Nucleic Acids Res 2014, 42(1):D780-D788.

14. Hu Y, Roesel C, Flockhart I, Perkins L, Perrimon N, Mohr SE: UP-TORR: online tool for accurate and Up-to-Date annotation of RNAi Reagents. Genetics 2013, 195(1):37-45.
15. Kulathinal RJ: Functional genetics in the post-genomics era: building a better roadmap in Drosophila. Genetics 2013, 195(1):7-8.

16. Hurov KE, Cotta-Ramusino C, Elledge SJ: A genetic screen identifies the Triple T complex required for DNA damage signaling and ATM and ATR stability. Genes Dev 2010, 24(17):1939-1950.

17. Tsui M, Xie T, Orth JD, Carpenter AE, Rudnicki S, Kim S, Shamu CE, Mitchison TJ: An intermittent live cell imaging screen for siRNA enhancers and suppressors of a kinesin-5 inhibitor. PLoS One 2009, 4(10):e7339.

18. Schultz N, Marenstein DR, De Angelis DA, Wang WQ, Nelander S, Jacobsen A, Marks DS, Massague J, Sander C: Off-target effects dominate a largescale RNAi screen for modulators of the TGF-beta pathway and reveal microRNA regulation of TGFBR2. Silence 2011, 2:3.

19. Smith JA, White EA, Sowa ME, Powell ML, Ottinger M, Harper JW, Howley PM: Genome-wide siRNA screen identifies SMCX, EP400, and Brd4 as E2-dependent regulators of human papillomavirus oncogene expression. Proc Natl Acad Sci U S A 2010, 107(8):3752-3757.

20. Grueneberg DA, Degot S, Pearlberg J, Li W, Davies JE, Baldwin A, Endege W, Doench J, Sawyer J, Hu Y, Boyce F, Xian J, Munger K, Harlow E: Kinase requirements in human cells: I. Comparing kinase requirements across various cell types. Proc Natl Acad Sci U S A 2008, 105(43):16472-16477.

21. Moffat J, Grueneberg DA, Yang X, Kim SY, Kloepfer AM, Hinkle G, Piqani B, Eisenhaure TM, Luo B, Grenier JK, Carpenter AE, Foo SY, Stewart SA, Stockwell BR, Hacohen N, Hahn WC, Lander ES, Sabatini DM, Root DE: A lentiviral RNAi library for human and mouse genes applied to an arrayed viral high-content screen. Cell 2006, 124(6):1283-1298.

22. Luo $B$, Cheung HW, Subramanian A, Sharifnia T, Okamoto M, Yang X, Hinkle G, Boehm JS, Beroukhim R, Weir BA, Mermel C, Barbie DA, Awad T, Zhou X, Nguyen T, Piqani B, Li C, Golub TR, Meyerson M, Hacohen N, Hahn WC, Lander ES, Sabatini DM, Root DE: Highly parallel identification of essential genes in cancer cells. Proc Natl Acad Sci U S A 2008, 105(51):20380-20385.

23. Cheung HW, Cowley GS, Weir BA, Boehm JS, Rusin S, Scott JA, East A, Ali LD, Lizotte PH, Wong TC, Jiang G, Hsiao J, Mermel CH, Getz G, Barretina J, Gopal S, Tamayo P, Gould J, Tsherniak A, Stransky N, Luo B, Ren Y, Drapkin R, Bhatia SN, Mesirov JP, Garraway LA, Meyerson M, Lander ES, Root DE, Hahn WC: Systematic investigation of genetic vulnerabilities across cancer cell lines reveals lineage-specific dependencies in ovarian cancer. Proc Natl Acad Sci U S A 2011, 108(30):12372-12377.

24. Staller MV, Yan D, Randklev S, Bragdon MD, Wunderlich ZB, Tao R, Perkins $L A$, Depace $A H$, Perrimon N: Depleting gene activities in early Drosophila embryos with the "maternal-Gal4-shRNA" system. Genetics 2013, 193(1):51-61.

25. Yan D, Neumüller RA, Buckner M, Ayers K, Li H, Hu Y, Yang-Zhou D, Pan L, Wang X, Kelley C, Vinayagam A, Binari R, Randklev S, Perkins LA, Xie T, Cooley $L$, Perrimon N: A regulatory network of Drosophila germline stem cell self-renewal. Dev Cell 2014, 28(4):459-473.

26. Ni JQ, Zhou R, Czech B, Liu LP, Holderbaum L, Yang-Zhou D, Shim HS, Tao R, Handler D, Karpowicz P, Binari R, Booker M, Brennecke J, Perkins LA, Hannon GJ, Perrimon N: A genome-scale shRNA resource for transgenic RNAi in Drosophila. Nat Methods 2011, 8(5):405-407.

doi:10.1186/1471-2105-15-192

Cite this article as: Yilmazel et al: Online GESS: prediction of miRNA-like off-target effects in large-scale RNAi screen data by seed region analysis. BMC Bioinformatics 2014 15:192.

\section{Submit your next manuscript to BioMed Central and take full advantage of:}

- Convenient online submission

- Thorough peer review

- No space constraints or color figure charges

- Immediate publication on acceptance

- Inclusion in PubMed, CAS, Scopus and Google Scholar

- Research which is freely available for redistribution 